\title{
Cell-Centric and User-Centric Multi-User Scheduling in Visible Light Communication aided Networks
}

\author{
Xuan $\mathrm{Li}^{1}$, Rong Zhang ${ }^{1}$, Jiaheng Wang ${ }^{2}$, Lajos Hanzo ${ }^{1}$ \\ ${ }^{1}$ Communications, Signal Processing and Control, School of ECS, University of Southampton, SO17 1BJ, UK \\ ${ }^{2}$ National Mobile Communications Research Laboratory, Southeast University, Nanjing, 210096, China
}

\begin{abstract}
Visible Light Communication (VLC) combined with advanced illumination has been expected to become an integral part of next generation heterogeneous networks at the time of writing, by inspiring further research interests. From both the Cell-Centric (CC) and the User-Centric (UC) perspectives, various VLC cell formations, ranging from fixed-shape regular cells with different Frequency Reuse (FR) patterns and merged cells employing advanced transmission scheme to amorphous userspecific cells are investigated. Furthermore, different Multi-User Scheduling (MUS) algorithms achieving Proportional Fairness (PF) are implemented according to different cell formations. By analysing some critical and unique characteristics of VLC, our simulation results demonstrate that, the proposed MUS algorithms are capable of providing a high aggregate throughput and achieving modest fairness with low complexity in most of the scenarios considered.
\end{abstract}

\section{INTRODUCTION}

With the promise of gaining access to a huge unlicensed bandwidth, which is available in the optical domain of the electromagnetic spectrum, Visible Light Communication (VLC) using commercially available Light-Emitting Diode (LED) transmitters offers a huge data rate potential in this licensefree spectral domain, whilst simultaneously satisfying energyefficient illumination demands. Extensive investigations have been dedicated to the VLC networks [1]-[7], as also indicated by the IEEE 802.15 .7 standard ratified for short-range visible light wireless communication [8].

Apart from the multi-fold advantages, naturally, VLC systems may exhibit some potential drawbacks, such as for example: 1) VLC networks perform poorly in non-line-ofsight scenarios owing to the predominantly Line-Of-Sight (LOS) propagation of light; 2) VLC networks fail to provide convenient Up-Link (UL) coverage at the current-state-of-theart; 3) In VLC networks, each optical Access Point (AP) illuminates only a small confined cell compared to cellular RF networks. In order to improving the performance of VLC networks, we study various VLC cell formation designs. From the Cell-Centric (CC) perspective, fixed-shape regular cells with different Frequency Reuse (FR) patterns and merged cells employing advanced transmission scheme are investigated. On the other hand, we propose the amorphous user-specific cells design from the User-Centric (UC) perspective.

Furthermore, when multiple users are present in the VLC network, fair and efficient Multi-User Scheduling (MUS) constitutes one of the significant problems, which in fact affects all multi-user networks. However, the MUS problem of VLC-based networks has remained to a large extent hitherto unexplored owing to the paucity of literature, especially when combined with various VLC cell formations. Against the above-mentioned background, in this paper, we investigate the MUS problem relying on the users' Proportional Fairness $(P F)$ as a measure, when jointly considering various VLC cell formations, ranging from fixed-shape regular cells with different Frequency Reuse (FR) patterns and merged cells employing advanced transmission scheme to amorphous userspecific cells. Both the Cell-Centric (CC) and the User-Centric (UC) scheduling algorithms are proposed, which perform efficiently with low complexity.

The rest of this paper is organized as follows. Our system model and the various cell formations considered are presented in Section II. Our methodology for scheduling users, including both the $\mathrm{CC}$ as well as the UC algorithms are described and evaluated in Section III and Section IV, respectively. Finally, Section V offers our conclusions.

\section{System MOdEL}

A VLC Down-Link (DL) network is considered, which is constituted by a set of VLC APs. More explicitly, each VLC AP relies on an LED lamp constructed from several LEDs. Before investigating our MUS problem, let us first discuss a range of VLC cell formations from both the cells' and the users' perspectives, i.e. CC formation and UC formation, respectively.

\section{A. Link characteristic}

Since each user has a limited Field-Of-View (FOV), they can only receive information from the optical APs, when one or more APs reside within the user's FOV. According to [9], if the angle of incidence $\psi$ from an AP to a user is less than the user's FOV $\psi_{\mathrm{F}}$, the optical channel's total Direct Current (DC) attenuation is given by

$$
H=\frac{(m+1) D_{\mathrm{PA}}}{2 \pi r^{2}} \cos ^{m}(\phi) T_{s}(\psi) g(\psi) \cos (\psi),
$$

where the Lambert index $m$ depends on the semi-angle $\phi_{1 / 2}$ at half-illuminance of the source, which is given by 
TABLE I: VLC Parameters

\begin{tabular}{l|r}
\hline \hline Transmitted optical power per LED lamp $\left(P_{t}\right)$ & $20[\mathrm{~W}]$ \\
Semi-angle at half power $\left(\phi_{1 / 2}\right)$ & $60^{\circ}$ \\
Modulation bandwidth $(B)[6]$ & $20[\mathrm{MHz}]$ \\
Physical area of a PD $\left(D_{\mathrm{PA}}\right)$ & $1.0\left[\mathrm{~cm}^{2}\right]$ \\
Gain of an optical filter $\left(T_{s}(\psi)\right)$ & 1.0 \\
Refractive index of a lens at a PD $(n)$ & 1.5 \\
O/E conversion efficiency $(\gamma)$ & $0.53[\mathrm{~A} / \mathrm{W}]$ \\
Half of the receiver's FOV $\left(\psi_{\mathrm{F}}\right)$ & $60^{\circ} / 62.5^{\circ}$ \\
BER threshold & $10^{-5}$ \\
\hline \hline
\end{tabular}

$m=-1 / \log _{2}\left(\cos \phi_{1 / 2}\right) . D_{\mathrm{PA}}$ is the detector's physical area for a Photo-Diode (PD), $r$ is the distance between the VLC transmitter and the receiver. Still referring to $(1), T_{s}(\psi)$ and $g(\psi)$ denote the gain of the optical filter and of the optical concentrator employed, respectively, while $g(\psi)$ can be written as $g(\psi)=n^{2} / \sin ^{2} \psi_{\mathrm{F}}$ [9], where $n$ is the refractive index of a lens at a PD. The average received power including all reflections may be negligible compared with the direct received average power of the LOS path. Therefore we may ignore the reflected optical power for simplicity and consider only the LOS-power as the desired received power. Our parameter values are summarized in TABLE I.

Furthermore, let us define the Signal to Interference plus Noise Ratio (SINR) as the aggregate electronic power received from signal set $\boldsymbol{S}_{S} \subseteq \boldsymbol{S}$, where $\boldsymbol{S}$ denotes the set of all the corresponding transmitting VLC APs, over the noise power in a bandwidth of $B$ [MHz] [6] plus the sum of the electronic power received from other optical sources in interference set $\boldsymbol{S}_{I}$, which is the complementary set of $\boldsymbol{S}_{S}$. Since the corresponding electronic power is proportional to the square of the amplitude of the electronic current, we can write the SINR as

$$
\xi=\frac{\gamma^{2} \sum_{i \in \boldsymbol{S}_{S}} P_{r, i}^{2}}{N_{0} B+\gamma^{2} \sum_{i \in \boldsymbol{S}_{I}} P_{r, i}^{2}},
$$

where $N_{0}\left[\mathrm{~A}^{2} / \mathrm{Hz}\right]$ is the noise power spectral density dominated by the shot noise $N_{\text {shot }}$ [7], given as $N_{0} \cong N_{\text {shot }}=$ $q I_{a}\left(P_{r}\right) \sim 10^{-22}$, where $q$ denotes the electron charge and $I_{a}\left(P_{r}\right)$ is the photo-current at the receiver [6]. The expression in (2) is in its common form and it will be different for each of the VLC cell formations, which are discussed next.

\section{B. Regular Cells}

1) Unity Frequency Reuse: As shown in Figure 1a, each VLC AP illuminates an individual cell and reuse the same frequency $f$ across all cells, which is referred to as the Unity Frequency Reuse (UFR) design. The shaded areas represent the Inter-Cell Interference (ICI) imposed by the LOS ray conveying different information and arriving from the neighbouring cells at the cell edge. For the triangular point shown in Figure 1a, ICI arrives from all the other three neighbouring cells in this scenario. If the FOV was sufficiently narrow, the
ICI may be mitigated since the user can only receive data from a single VLC AP. However, this would potentially lead to isolated 'coverage islands' and 'coverage holes', which consequently may result into frequent horizontal handovers and outage event, when the DL user is walking between VLC APs, since the user will experience dramatic performance degradation in the area without LOS coverage.

2) Non-unity Frequency Reuse: Following the traditional cellular design principle, non-unity Frequency Reuse (FR) patterns may be employed for reducing the ICI, while each VLC AP still functions as an individual cell. Since the firsttier neighbouring cells contribute most of the ICI, while the second-tier cells generally have a negligible influence, an FR factor of two may be used. For the triangular point shown in Figure $1 b$, the ICI emanating from the neighbouring cells $B$ and $C$ can be removed. Although this is an appealingly simple solution, when using an FR factor larger than one, the system has to obey the classic trade-off between reduced Bandwidth Efficiency (BE) and improved cell-edge SINR. In fact, supporting mobility is the most grave problem associated with non-unity FR during VLC cell formation, since switching between frequencies every few meters during the user's movement degrades the user experience. This is also the reason for not considering FFR [5], which exhibits a more elaborate frequency planning and triggers even more frequent handovers.

\section{Merged Cells}

In order to reduce the size of the ICI-infested areas, whilst improving the mobility, several neighbouring cells can be merged into a large multi-AP cell, where advanced transmission techniques may be employed in their overlapping areas. In the following, we use UFR across multi-AP cells for simplicity, although non-unity FR might be also used.

1) Combined Transmission: In this arrangement, each individual VLC AP of a multi-AP cell conveys the same information on the same visible carrier frequency in their overlapping areas. In Figure 1c, $A$ and $B$ are merged into a 2-AP cell and transmit identical signals in their overlapping area as a single source, which we refer to as Combined Transmission (CT). Thus the potential ICI is beneficially turned into useful signals which may be combined and the original cell edges of Figure 1a become the cell centres of Figure 1c. Although the SINR may be enhanced, $\mathrm{CT}$ results in a reduced $\mathrm{BE}$, since only a single user is served at a time by several APs in the overlapping area within a merged cell.

2) Vectored Transmission: In order to eliminate the BEreduction imposed by $\mathrm{CT}$, Zero-Forcing (ZF)-based Vectored Transmission (VT) techniques can be employed for serving multiple users at the same time in the overlapping area. The underlying principle of ZF-based VT is to totally eliminate the ICI at the multiple transmitters, so that the multiple users receive mutually interference-free signals. In general, to facilitate VT from $n_{\alpha}$ APs to $U$ users, both the $\left(U \times n_{\alpha}\right)$ element DC attenuation matrix and the users' data have to be shared amongst the $n_{\alpha}$ APs [10]. For VLC channels, the 


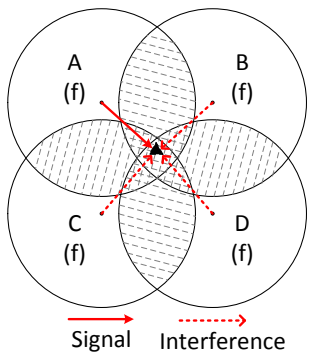

(a) UFR

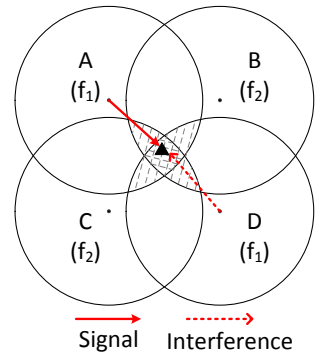

(b) FR-2

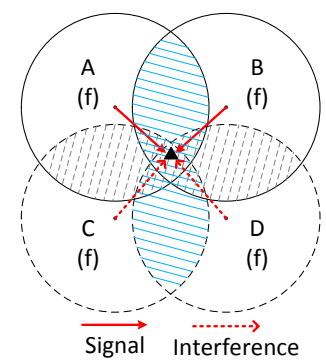

(c) CT-2

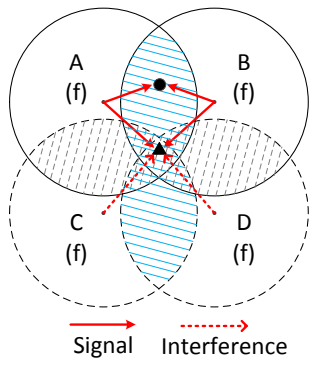

(d) VT-2

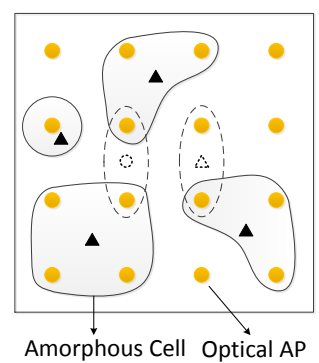

(e) UC

Fig. 1: Different cell formations. (a) is a regular cell formation with UFR, (b) has a FR factor of two, (c) represents two merged 2-AP cells with CT, (d) shows two merged 2-AP cells using VT and (e) represents the UC amorphous cell formations. The triangle and circle denote certain points of reception. In (a)-(d), the shaded areas covered with dotted lines represent the ICI imposed by the LOS ray of neighbouring cells at the cell edge and the shaded areas covered by solid lines represent the overlapping areas within the merged 2-AP cells. In (a), (c), (d) and (e), the entire frequency band $f$ is used by each small cell, while in (b) orthogonal frequencies $f_{1}$ and $f_{2}$ are employed by neighbouring cells, where we have $f_{1}=f_{2}=f / 2$.

requirements may be readily satisfied, since the VLC users are pre-predominantly stationary.

\section{User-Specific Cells}

Either the above-mentioned regular cells or merged cells are pre-set by the system with fixed shape regardless of the traffic requirements, which is referred to as the $\mathrm{CC}$ formation. Against the $\mathrm{CC}$ fixed-shape cell formation designs, the proposed $U C$ design is capable of supporting irregular-shape elastic cell formations according to dynamic traffic requirements. In our UC design, amorphous cells are constructed without a clear cell boundary, since the user-specific AP combinations are of different cardinality. Furthermore, they are updated, when the users move, join or leave the system. This leads to flexible, 'breathing' and evolving cells [1].

The UC design using VT may be expected to provide a higher BE, but if the complexity was unaffordable, a potential simplification is to invoke interference avoidance for the amorphous cells. As an example in Figure 1e, the triangle point with dashed boundary and the triangle point on the bottom right can receive information from the same AP. Instead of employing the sophisticated ZF-based VT and serving them simultaneously, the proposed UC-based MUS algorithm grants access for one of them at a time and allocates all the corresponding transmitting APs for jointly serving this active point at any instant. Thus the ICI can be eliminated. Still referring to Figure 1e, the dashed circle point and its two related triangle points are scheduled under the abovementioned rule as well. All the disjoint groups in Figure 1e are scheduled in a parallel manner upon using our scheduling algorithm, which will be mathematically detailed in Section III.

\section{Methodology}

In this section, we commence with a general formulation of our MUS problem and then come to the $\mathrm{CC}$ and UC sce- narios, respectively. Furthermore, we propose our CC and UC scheduling algorithms for determining how to serve users ${ }^{1}$.

\section{A. General Problem Formulation}

Our aim is to find the optimal resource allocation using the Objective Function (OF) of maximizing the long-term network wide utility, while schedule the users in a PF manner, which is ultimately a joint cell formation and MUS problem. We would like to introduce some notations first. Let $\mathcal{N}$ be the set of all APs in the system model and $\mathcal{U}$ be the set of all users that are assumed to be uniformly distributed at random in this VLC system. We have $|\mathcal{N}|=N$ and $|\mathcal{U}|=U$. Furthermore, $\mathrm{C}_{\mu}$ denotes the cell serving the user $\mu$, which is constituted by several APs among $\mathcal{N}$. If the user $\mu$ is not being served, $\mu$ is regarded as inactive and we have $\mathcal{C}_{\mu}=\emptyset$. Let $r_{\mu}^{(t)}$ be the achievable data rate of the user $\mu$ at time slot $t$ and the calculation of $r_{\mu}^{(t)}$ will be detailed in Section IV.

Upon adopting a PF scheduler [11], our joint problem may generally be formulated as:

$$
\max . \quad \sum_{\mu \in \mathcal{U}} \frac{r_{\mu}^{(t)}}{\hat{R}_{\mu}^{(t)}}, \quad \text { s.t. } \quad \mathcal{C}_{\mu} \subseteq \mathcal{N}, \forall \mu \in \mathcal{U},
$$

where $\hat{R}_{\mu}^{(t)}$ denotes the long-term average throughput at slot $t$. According to [12], $\hat{R}_{\mu}^{(t)}$ may be obtained over a time window as a moving average:

$$
\hat{R}_{\mu}^{(t)}=\left\{\begin{array}{ll}
\left(1-\frac{1}{T_{\mathrm{F}}}\right) \hat{R}_{\mu}^{(t-1)}+\frac{1}{T_{\mathrm{F}}} r_{\mu}^{(t)} & \text { if scheduled } \\
\left(1-\frac{1}{T_{\mathrm{F}}}\right) \hat{R}_{\mu}^{(t-1)} & \text { if not scheduled }
\end{array},\right.
$$

where $\hat{R}_{\mu}^{(t-1)}$ denotes the long-term average throughput at slot $(t-1)$ and $T_{\mathrm{F}}$ is the length of the time window. (3) is

\footnotetext{
${ }^{1}$ The implementation of our proposed scheduling algorithms may rely on a decoupled data and control system, where the control function is assigned to an umbrella Radio Access Technology (RAT) over-sailing above the VLC cells, which is detailed in [1].
} 
the general formulation of the joint problem and the different formulations for various cell formations will be discussed next.

\section{B. Cell-Centric Scheduling}

1) Formulation: The $\mathrm{CC}$ cell formation designs, ranging from the regular cells to the merged cells, support pre-set fixed-shape optical cells. Hence, $\mathcal{C}_{\mu}$ only have several fixed constitution, which is denoted as $\mathcal{C}_{\text {set }}$. (3) may be written as:

$$
\begin{array}{cll}
\max . & \sum_{\mu \in \mathcal{U}} \frac{r_{\mu}^{(t)}}{\hat{R}_{\mu}^{(t)}}, & \\
\text { s.t. } & I_{\mu}\left(\mathcal{C}_{1}, \cdots, \mathcal{C}_{\mu}\right), & \forall \mu \in \mathcal{U}, \\
& \mathcal{C}_{\mu} \subseteq \mathcal{C}_{\text {set }}, & \forall \mu \in \mathcal{U} .
\end{array}
$$

The constraint (6) indicates that for a certain $\mathrm{CC}$ formation, each user's ICI can be calculated according to the pre-set cell formation and the user's location. In (7), for any user $\mu$, its corresponding cell $\mathcal{C}_{\mu}$ should be included in the pre-set cells set $\mathcal{C}_{\text {set }}$.

2) Scheduling: For the purpose of scheduling users in a PF manner, let each cell schedule the user with the largest priority $r_{\mu}^{(t)} / \hat{R}_{\mu}^{(t)}$ within its coverage at slot $t$. Thus (5) is maximized. There is a special scenario. When two cells $\mathcal{C}_{i}$ and $\mathcal{C}_{j}$ are within the FOV of a single user $\mu, \mu$ may have the largest priority for both $\mathcal{C}_{i}$ and $\mathcal{C}_{j}$ and receive the connection request from both of them, but only one can serve $\mu$ under our assumption. Then $\mu$ will send back a relevant signal to $\mathcal{C}_{i}$ and $\mathcal{C}_{j}$ and a small-scale comparison will be made. Considering the users $i$ and $j$ with the second largest priority in these two cells, respectively, if $\left(r_{\mu}^{(t)} / \hat{R}_{\mu}^{(t)}+r_{i}^{(t)} / \hat{R}_{i}^{(t)}\right) \geq\left(r_{\mu}^{(t)} / \hat{R}_{\mu}^{(t)}+\right.$ $\left.r_{j}^{(t)} / \hat{R}_{j}^{(t)}\right), \mu$ connecting with $\mathcal{C}_{j}$ provides a higher utility for the system; otherwise, $\mu$ should be served by $\mathcal{C}_{i}$. After this comparison, $\mathcal{C}_{i}$ and $\mathcal{C}_{j}$ exchange information and decide which cell to connect with $\mu$. Let us now provide an overview of the scheduling algorithm in form of Algorithm 1 , where $\mathcal{U}_{e}$ denotes the set of all users within the coverage of the pre-set cell $\mathrm{C}$.

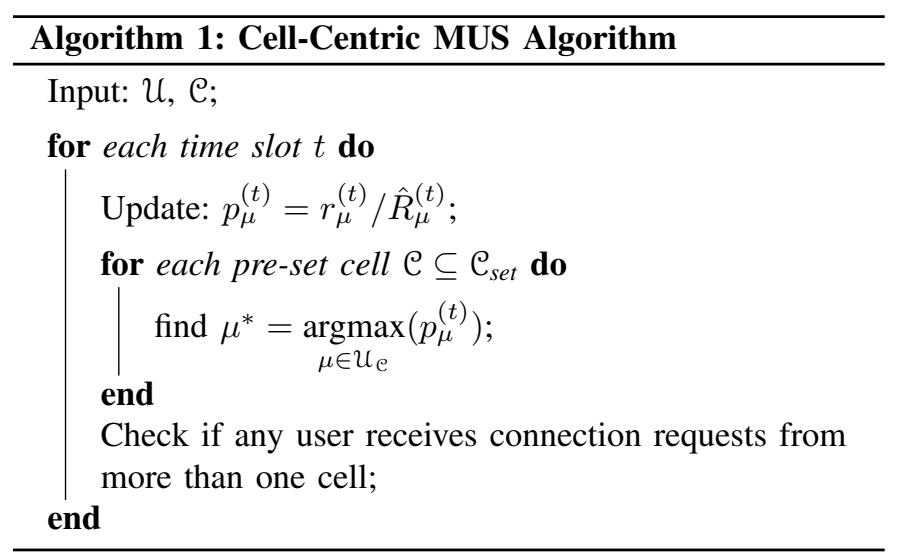

\section{User-Centric Scheduling}

1) Formulation: In this arrangement, the VLC cells are formed according to the users' conditions. Firstly, since we do not impose any constraints on the total number of the user-specific cells or the number of APs within a single cell, there are numerous ways to construct cells. Furthermore, the ICI received by each user can be determined only after all cells are formed. However, the ICI will reversely influence the users' data rate and furthermore the solution for (3). Listing all possible amorphous cell formations is one of the approaches of finding the solution for our joint problem, while this may not be realistic, especially when the network is large-scale. Hence, we opt for a specific solvable scenario, where the interference is restricted to be zero for all active users. More explicitly, if two users are capable of receiving the data from the same AP $n$, they are referred to as neighbours and there may be potential interference. Since a single AP can only connect with one user at a slot, if the neighbouring users are both active, one of them will be served by $n$ and the other will receive ICI imposed by $n$. Thus, (3) may be written as:

$$
\begin{array}{cl}
\max . & \sum_{\mu \in \mathcal{U}} \frac{r_{\mu}^{(t)}}{\hat{R}_{\mu}^{(t)},} \\
\text { s.t. } & I_{\mu}=0, \\
& \mathcal{C}_{i} \cap \mathcal{C}_{j}=\emptyset, \quad \forall \mu \in \mathcal{U},
\end{array}
$$

(10) indicates that for any two users $i$ and $j$, the corresponding cells $\mathcal{C}_{i}$ and $\mathcal{C}_{j}$ cannot contain the same AP at the scheduling time slot $t$. To elaborate a little further, in Figure 1e, the circle point with dashed boundary has two triangle points as neighbours. Under the constraint of (10), the circle's cell with the dashed boundary and its two neighbours' cells with solid boundary cannot exist at the same time, since they have the same APs as intersection. More explicitly, either the circle or its two neighbours are served at a single time slot. Thus the ICI is totally eliminated. In a parallel manner, other neighbour groups are also scheduled under the same constraint.

2) Scheduling: (i) Graph Model: An efficient solution of (8) on the basis of graph theory and the minimum-degree greedy algorithm [13] is proposed in this section. Some notations are defined first. Let $\mathcal{G}(\mathcal{V}, \mathcal{E})$ denote a graph with the vertex set $\mathcal{V}(\mathcal{G})$ and the edge set $\mathcal{E}(\mathcal{G}) . \mathcal{V}(\mathcal{G})$ is the user set $U$ and a vertex in $\mathcal{V}(\mathcal{G})$ is denoted by $v_{\mu}$, which matches a certain user in our system. The edge set $\mathcal{E}(\mathcal{G})$ denotes the relationship between users. We then introduce an edge between neighbouring vertices. Let $\mathcal{D}\left(v_{\mu}\right)$ denote the neighbouring vertices and its cardinality $d\left(v_{\mu}\right)$ may be seen as the degree of $v_{\mu}$. Next we will add a weight to each vertex and the graph model may be updated to a weighted graph $\mathcal{G}^{w}(\mathcal{V}, \mathcal{E})$. The mechanism of PF scheduler is to schedule the user with the largest maximum priority $p_{\mu}$ at each time slot, where $p_{\mu}=r_{\mu}^{(t)} / \hat{R}_{\mu}^{(t)}$. Thus the priority of each user may be interpreted as the weight of each vertex in $\mathcal{G}^{w}(\mathcal{V}, \mathcal{E})$. The problem (8)-(10) may be transformed into the following problem in a weighted interference graph: find a vertices set with the maximum sum weight excluding any edges. This problem is a Maximum Weighted Independent Set Problem (MWISP), which may be NP-complete and consume unacceptable time 
to solve. Thus we opt for a heuristic greedy algorithm, which proves to be fast and efficient in [13].

(ii) Scheduling algorithm: Next, we will introduce our heuristic algorithm for scheduling users in a PF manner. Upon using the MIN greedy algorithm, the vertex with the smallest degree will be scheduled at each slot. Considering the weight of each vertex, $p_{\mu} / d\left(v_{\mu}\right)$ may be regarded as the weighted priority of the user $\mu$. At each slot, the central manager schedule the user (vertex) with the highest priority and then remove it and its neighbours from the current graph. Then the graph is upgraded and the central manager repeats the scheduling process until no vertex in the graph. Furthermore, at the beginning of each time slot, the controller first checks isolated vertices without a neighbour, which are supposed to be scheduled at every slot, provided that there are one or more APs within the FOV. Thus the isolated vertices are granted access but not be considered by the scheduling process. Note that in order to avoid ICI, when a user is scheduled, all the APs within its FOV should employ CT conveying identical data for this user. Let us now provide an overview of the scheduling algorithm in form of Algorithm 2.

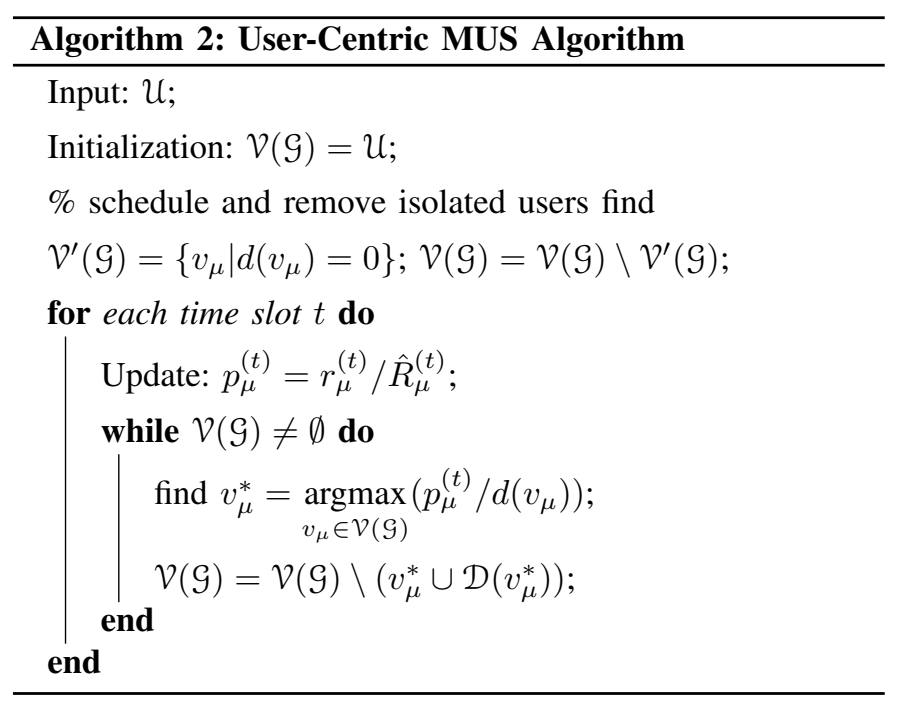

\section{EVALUATIONS}

In this section, we will present our simulation results for our scheduling algorithms with special emphasis on the aforementioned various VLC cell formations. A $15 \mathrm{~m} \times 15 \mathrm{~m} \times 3 \mathrm{~m}$ room model is considered, which is covered by a VLC system including $4 \times 4$ uniformly distributed optical APs at a height of $2.5 \mathrm{~m}$. The parameters of the LED lamps are summarized in TABLE I. For simplicity, we consider baseband transmissions without subcarrier modulation at this stage. Pulse-Amplitude Modulation having an order of $M(M$-PAM) is used. Based on our Bit Error Rate (BER) performance results, given a certain target BER, Signal to Noise Ratio (SNR) and Signal to Interference Ratio (SIR), the maximum affordable $M$ PAM order capable of maintaining the target-BER can be determined. Thus the attainable throughput between the AP
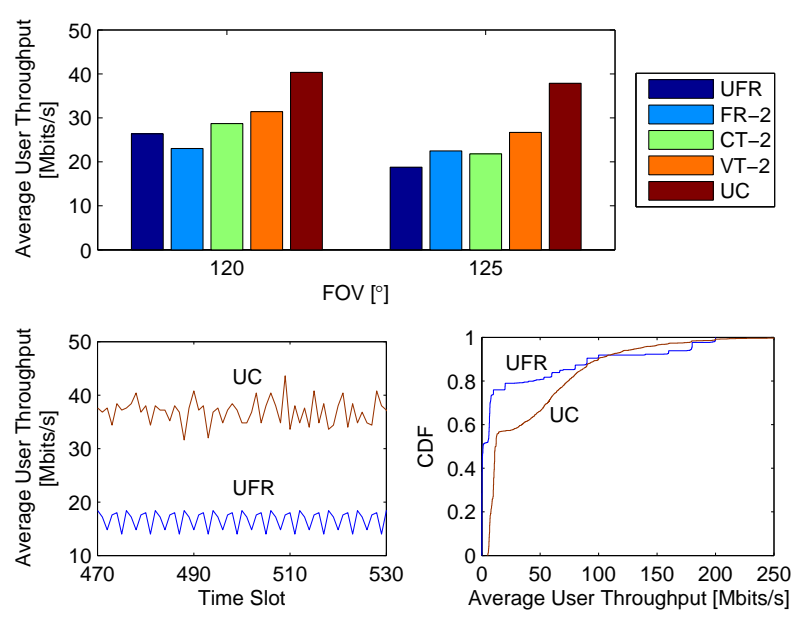

Fig. 2: MUS performance of our VLC networks with various cell formation scenarios. The top is the average user throughput under different FOV. The bottom left records the average user throughput between the 470th to the 530th time slots in the 25th simulation snapshot and the bottom right illustrates the Cumulative Distribution Function (CDF) of user throughput for the UFR and the UC cell formation scenarios.

$n$ and the user $\mu$ becomes $r_{n \mu}=2 B \log _{2} M /(1+\rho)$, where the roll-off factor of the raised-cosine pulses is assumed to be $\rho=1$. Our simulation results are averaged over 50 independent snapshots and each snapshot contains 1,000 time slots.

FOV is one of the factors that is expected to significantly influence the ICI in VLC-based networks. Increasing the FOV leads to the expansion of the ICI-contaminated areas and correspondingly the employment of ICI reduction techniques may become more important. Figure 2 shows the system's performance by employing different cell formation designs after performing the MUS algorithms. The top is the average user throughput under different FOV, which suggests that as expected, the throughput provided by the UC cells is higher than the other cell formations for both FOV values. The bottom left records the average user throughput between the 470th to the 530th time slot in the 25th simulation snapshot and the bottom right illustrates the Cumulative Distribution Function (CDF) of user throughput for the UFR and the UC cell formation scenarios. Furthermore, in order to investigate the LOS characteristic of this VLC system, We introduce the LOS blocking probability $P_{\text {block }}$ to represent the probability that the LOS VLC path is blocked, which may lead to a reduction of the data rate experienced by some VLC users. Then the VLC DL data rate becomes $\widetilde{\mathrm{R}}=P_{\text {block }} \cdot 0+\left(1-P_{\text {block }}\right) \cdot \mathrm{R}$. At this stage, we assume that all LOS paths are blocked with an equal probability. Figure 3 indicates that, the average user throughput is reduced upon increasing the LOS blocking probability in all cell formation scenarios.

We will use the Service Fairness Index (SFI) of [14] to reflect the grade of fairness experienced by the users. The 


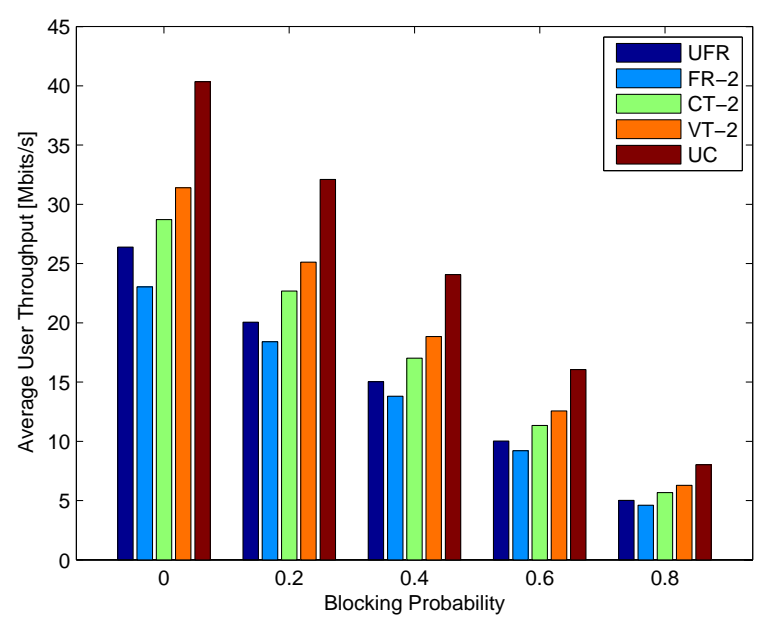

Fig. 3: Average user throughput under various LOS blocking probabilities, $\mathrm{FOV}=120^{\circ}$.

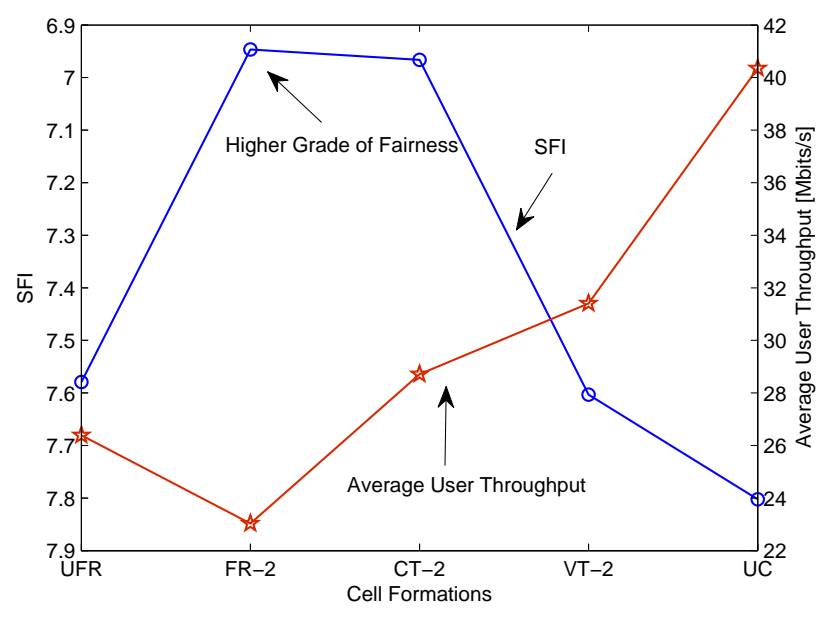

Fig. 4: The SFI and average user throughput, FOV $=120^{\circ}$. The grade of fairness perceived is reduced upon increasing the SFI value.

objective of ensuring fairness amongst the users is to guarantee that all users benefit from the same throughput within a given period, provided that the users' data rate requirements are identical. However, this is often unrealistic. The SFI of [14] is introduced to represent the difference between the maximum and the minimum amongst all the users' throughput. When the SFI is low, the throughput-difference of different users is small, hence they are served fairly. By contrast, if the SFI is high, the users experiencing a lower data rate may complain about their unfair treatment. Therefore a lower SFI means a higher grade of individual fairness. The specific SFI values of the different cell formation scenarios considered are plotted in Figure 4. It can be clearly seen that the users' throughputdifference is the smallest in the FR-2 scheme. By contrast, the UC design is capable of providing the highest throughput but its SFI is the lowest in the scenarios considered, which may be more attractive when considering users' multi-service requirements.

\section{CONCLUSions}

In this paper, various VLC cell formation schemes and the MUS for a DL system constituted by several VLC APs were investigated. We studied the regular design concept borrowed from cellular networks relying on different FR factors in VLC environments, merged multi-AP cells employing either CT or ZF-based VT and the user-specific amorphous cells. To solve the essential MUS problem in the context of our VLC system, both CC and UC algorithms were invoked for implementing a PF scheduler. We analysed the average user throughput and fairness of this VLC system. By employing the UC scheduling algorithm, the VLC network becomes capable of providing a higher throughput, while the throughput-difference of different users is the largest. This may provide some insights for this VLC systems' design, when various traffic requirements are considered.

\section{REFERENCES}

[1] R. Zhang, J. Wang, Z. Wang, Z. Xu, C. Zhao, and L. Hanzo, "Visible light communications in heterogeneous networks: pave the way for usercentric design," IEEE Wireless Communications (Accepted), 2014.

[2] X. Li, R. Zhang, and L. Hanzo, "Cooperative load balancing in hybrid visible light communications and wifi," IEEE Transactions on Communications (Accepted), 2014.

[3] F. Jin, R. Zhang, and L. Hanzo, "Resource allocation under delayguarantee constraints for heterogeneous visible-light and RF femtocell," IEEE Transactions on Wireless Communications (Accepted), 2014.

[4] C. Chen, D. Tsonev, and H. Haas, "Joint transmission in indoor visible light communication downlink cellular networks," in IEEE GLOBECOM Workshops 2013, Dec. 2013, pp. 1127-1132.

[5] C. Chen, N. Serafimovski, and H. Haas, "Fractional frequency reuse in optical wireless cellular networks," in IEEE PIMRC 2013, Sep. 2013 pp. 3594-3598.

[6] J. Grubor, S. Randel, K.-D. Langer, and J. Walewski, "Broadband information broadcasting using led-based interior lighting," Journal of Lightwave Technology, vol. 26, no. 24, pp. 3883-3892, Dec. 2008.

[7] T. Komine and M. Nakagawa, "Fundamental analysis for visible-light communication system using led lights," IEEE Transactions on Consumer Electronics, vol. 50, no. 1, pp. 100-107, Feb. 2004.

[8] "IEEE standard for local and metropolitan area networks-part 15.7: Short-range wireless optical communication using visible light," IEEE Std 802.15.7-2011, pp. 1-309, Sep. 2011.

[9] J. Kahn and J. Barry, "Wireless infrared communications," in Proceedings of the IEEE, vol. 85, no. 2, Feb. 1997, pp. 265-298.

[10] R. Zhang and L. Hanzo, "Cooperative downlink multicell preprocessing relying on reduced-rate back-haul data exchange," IEEE Transactions on Vehicular Technology, vol. 60, no. 2, pp. 539-545, Feb. 2011.

[11] H. Kim, K. Kim, Y. Han, and S. Yun, "A proportional fair scheduling for multicarrier transmission systems," in VTC2004-Fall, vol. 1, Sep. 2004, pp. 409-413.

[12] T. Bu, L. Li, and R. Ramjee, "Generalized proportional fair scheduling in third generation wireless data networks," in Proceedings of INFOCOM 2006, Apr. 2006, pp. 1-12.

[13] M. M. Halldórsson and J. Radhakrishnan, "Greed is good: Approximating independent sets in sparse and bounded-degree graphs," Algorithmica, vol. 18, no. 1, pp. 145-163, May 1997.

[14] B. Bensaou, D. H. K. Tsang, and K. T. Chan, "Credit-based fair queueing (CBFQ): a simple service-scheduling algorithm for packet-switched networks," IEEE/ACM Transactions on Networking, vol. 9, no. 5, pp. 591-604, Oct. 2001 